\title{
A Novel Impression Technique for Transforming an Acrylic Hybrid Prosthesis into a Metal Ceramic One Using Combined Analog and Digital Workflows
}

\author{
Hani Tohme ${ }^{1}$, Ghida Lawand ${ }^{2}$, Mohammed Akl ${ }^{3}$
}

\begin{abstract}
Aim and objective: This technique describes a novel method for converting an acrylic hybrid into metal-ceramic using a combined digital and analog workflow.

Background: Although the acrylic hybrid prosthesis treatment modality has been consistently used for decades, complications such as occlusal wear and tooth debonding have brought to light other alternative restorative materials such as the use of zirconia or a metal framework layered with ceramic. Developing a technique that allows clinicians to convert existing acrylic prostheses easily and conveniently into metal-ceramic ones would overcome many of the complications associated with current acrylic hybrids.

Technique: A combined digital and analog workflow are utilized to convert an acrylic hybrid into a metal-ceramic one by making a repositioning impression with the existing prosthesis maintaining the interface fit for the new prosthesis. The metal framework of the new prosthesis was designed and manufactured digitally. After layering the framework with ceramic, the prosthesis is evaluated for ideal fit, occlusion, and esthetics before delivery.

Conclusion: The combined analog and digital workflow for converting an acrylic hybrid to a metal-ceramic prosthesis through a pick-up impression reduces overall chairside time and provides a predictably fitting prosthesis.

Keywords: Clinical technique, Conversion, Digital dentistry, Hybrids, Implant dentistry, Verification jig.

International Journal of Prosthodontics and Restorative Dentistry (2021): 10.5005/jp-journals-10019-1327
\end{abstract}

\section{INTRODUCTION}

Today, edentulous oral rehabilitation options often gravitate on implants to provide comfortable and stable prostheses to the patient that may be fixed or removable in nature. ${ }^{1,2}$ Different patient outcomes were reported when comparing fixed to removable complete implant-supported prostheses; however, fewer impediments were noticed with the fixed option due to the alleviated chances of residual ridge resorption, decreased prosthetic maintenance, and increased patient compliance..$^{1-3}$ There are various types of fixed complete prostheses that are classified based on the presence of horizontal or vertical composite defects, available prosthetic space, and the nature of the opposing dentition. ${ }^{4,5}$ For cases of pronounced intra-arch space, moderate lack of lip support, and a high lip line, the metal acrylic complete fixed dental prosthesis, initially referred to as a hybrid prosthesis, was introduced. ${ }^{1}$ Metal acrylic hybrid prostheses gained popularity due to their low dynamic occlusal loading risks, esthetic outcomes, long survival rate, and low cost. ${ }^{6-8}$ However, despite the favorable results achieved with such prosthetic rehabilitation options, several mechanical and esthetic complications are frequently observed such as abrasion caused by wear, discoloration due to resin staining, and tooth debonding as a result of the reduced thickness of the acrylic resin available for retention over the metal structure beneath (Fig. 1). ${ }^{8-11}$

To circumvent this problem, ceramic layering on a metal framework was suggested in lieu of acrylic resin with denture teeth. This alternative provided higher functional and esthetic outcomes and was met with few biological and mechanical complications,
${ }^{1}$ Department of Removable Prosthodontics, Saint Joseph University, Beirut, Lebanon

${ }^{2}$ Department of Prosthodontics and Esthetic Dentistry, Saint Joseph University, Beirut, Lebanon

${ }^{3}$ Department of Restorative Sciences, University of Minnesota, Minneapolis, Minnesota, USA

Corresponding Author: Hani Tohme, Department of Removable Prosthodontics, Saint Joseph University, Beirut, Lebanon, Phone: +961 03307910, e-mail: hani@tohmeclinic.com

How to cite this article: Tohme H, Lawand G, AkI M. A Novel Impression Technique for Transforming an Acrylic Hybrid Prosthesis into a Metal Ceramic One Using Combined Analog and Digital Workflows. Int J Prosthodont Restor Dent 2021;11(3):138-141.

Source of support: Nil

Conflict of interest: None

as well as a high prevalence of implant and prosthesis survival. ${ }^{12}$ While some authors ${ }^{13}$ have attempted to use the patient's existing prosthesis as a guide to fabricate a new monolithic zirconia hybrid, the process still entailed making a final impression and pouring a master cast and relying on special cemented cylinders which, hypothetically, ensure a passively fitting final prosthesis. This novel technique describes a step-by-step impression procedure for obtaining immediate passive fit while transforming a metal acrylic hybrid prosthesis to a ceramic-layered hybrid prosthesis using combined conventional and digital workflows.

(o) The Author(s). 2021 Open Access This article is distributed under the terms of the Creative Commons Attribution 4.0International License (https://creativecommons. org/licenses/by-nc/4.0/), which permits unrestricted use, distribution, and non-commercial reproduction in any medium, provided you give appropriate credit to the original author(s) and the source, provide a link to the Creative Commons license, and indicate if changes were made. The Creative Commons Public Domain Dedication waiver (http://creativecommons.org/publicdomain/zero/1.0/) applies to the data made available in this article, unless otherwise stated. 


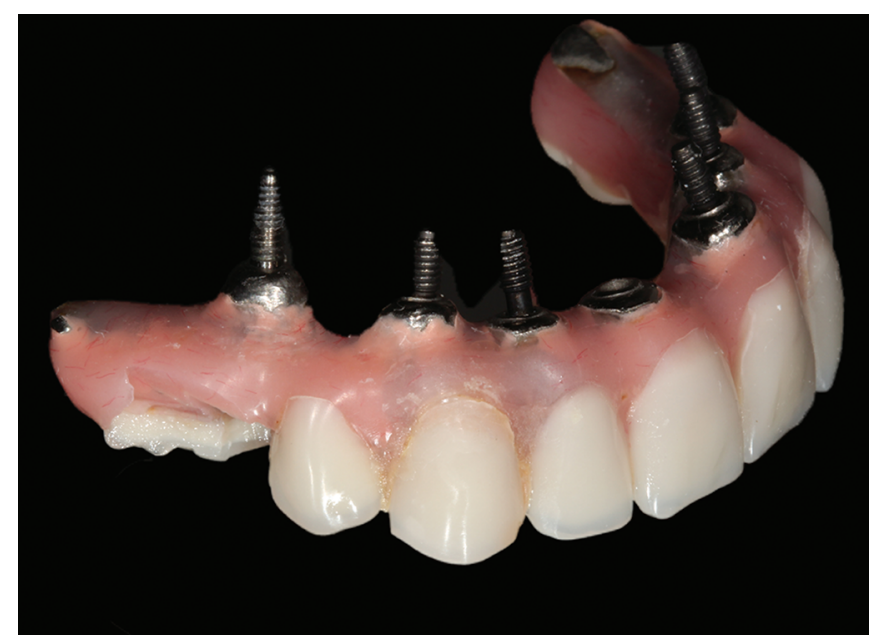

Fig. 1: Old fractured acrylic hybrid prosthesis

\section{TECHNIQUe}

- Obtain periapical radiographs with appropriate film positioners to ensure that the metal acrylic hybrid prosthesis is in intimate contact with the implants and that there are no complications. Perform a one screw test, where a single screw is tightened on the terminal abutment and the framework is evaluated on the contralateral side, to evaluate the existing prosthesis and ensure that it has an optimum, passive fit. It is also crucial to evaluate the prosthesis for occlusal wear or loss of occlusal vertical dimension before using it as a reference for esthetics or occlusion.

- Remove the composite and PTFE tape present in the access holes of the prosthesis and evaluate or re-establish the maintenance of preload at each implant site. Ensure that the screws are tightened well and torqued as per the manufacturer's instructions.

- Perform a "scan only" pre-preparation scan using an intraoral scanner (TRIOS 3; 3Shape, Copenhagen, Denmark) of the mandibular arch alone, the existing prosthesis alone (Fig. 2), and in occlusion. Export the files in a Standard Tessellation Language (STL) file format. These scans are necessary as a reference for the dental technician to design the metal framework.

- Mix putty consistency of polyvinyl siloxane (PVS) (Honigum Putty Rigid Fast; DMG Dental, Hamburg, Germany). Start by adopting the putty on the palatal side of the prosthesis and then move on to the buccal surface to ensure prosthesis stability in the impression material upon removal. Ensure that the PVS does not extend to and cover the screw access holes to allow for retrieval of the prosthesis. After the complete setting time of the PVS, as per the manufacturer's instructions, unscrew the prosthesis, and remove the impression of having picked up the hybrid within it (Fig. 3).

- Connect customized healing abutments to the implants intraorally to preserve the gingival soft tissue contours and prevent tissue displacement. If no custom healing abutments are available, light body PVS may be injected around prefabricated healing abutments to preserve soft tissue contours.

- Connect the corresponding implant analogs to the prosthesis then inject the gingival mask around the analogs (Gingifast Rigid; Zermach SpA, Italy). Pour the impression under a vibrator after mixing plaster powder (Convertin Hart; SpofaDental, KerrHawe,

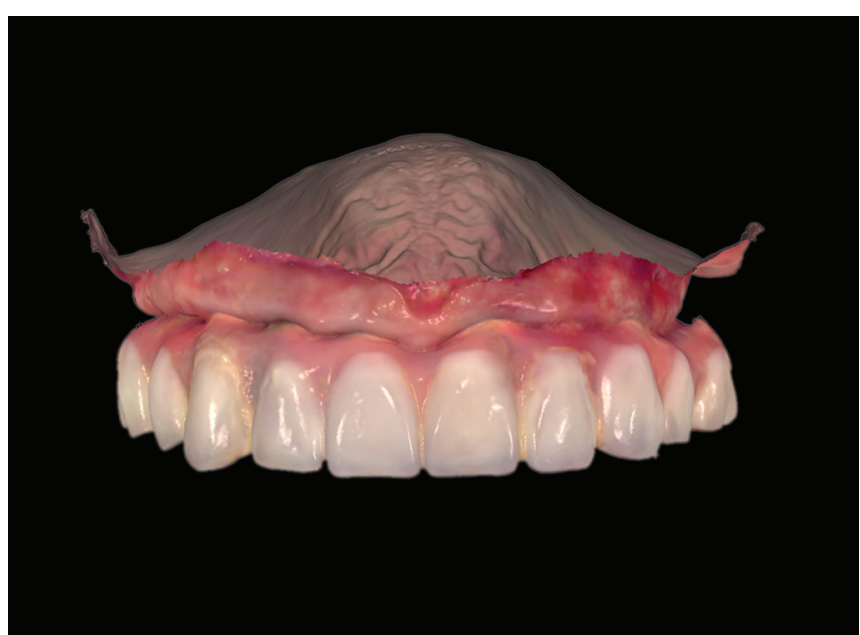

Fig. 2: Intraoral scan of the patient's maxillary arch with acrylic hybrid prosthesis inserted

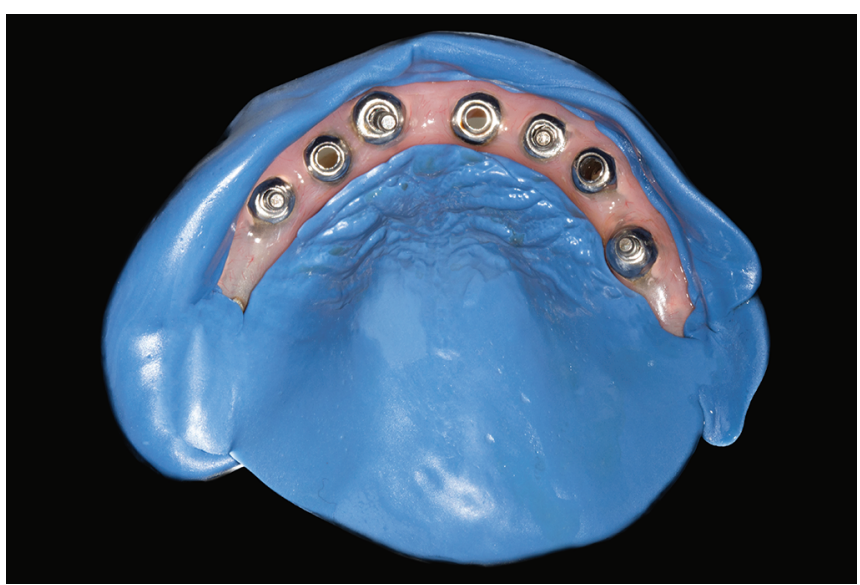

Fig. 3: Old prosthesis used to make a repositioning impression with putty consistency PVS. This gives the clinicians control of the material and allows them to keep it away from the screw access holes while adapting it

Bioggio, Switzerland) and distilled water following the ratio suggested by the manufacturer.

- Return the prosthesis into the patient's mouth and tighten the screws. Place PTFE tape followed by a provisional filling material (Cavit; 3M, Minnesota, USA) to seal the access holes temporarily.

- Scan the maxillary cast with and without scan bodies (Scanmarker; Zirkonzahn $\mathrm{GmbH}$, Gais, Italy) using a desktop scanner (S600 ARTI Scanner, Zirkonzahn GmbH, Gais, Italy). Import the scanned cast and the "scan only" STL files into the CAD software (Exocad Dental CAD; Exocad GmbH, Darmstadt, Germany) and superimpose the STLs using the incisive papilla and the palatal rugae as reference points.

- Design the new prosthesis in full contour using the previous prosthesis as a reference. Adjust your design as needed to improve your esthetic outcomes and then generate the design of the metal framework automatically by reducing the full contour design by 1.5 to $2 \mathrm{~mm}$ (Fig. 4).

- Export the STL file of the metal framework design and import it into a printing software (MTT AutoFab; Marcam Engineering $\mathrm{GmbH}$, Bremen, Germany) that will transform the STL file into an Advanced Forensics Format (AFF) file for printing. 


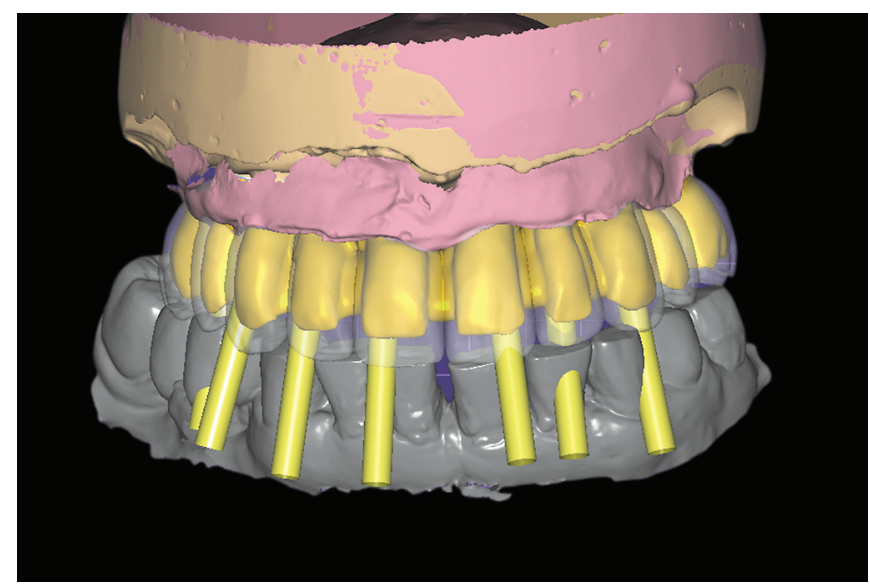

Fig. 4: Virtual reduction on Exocad software to create the design of the metal framework

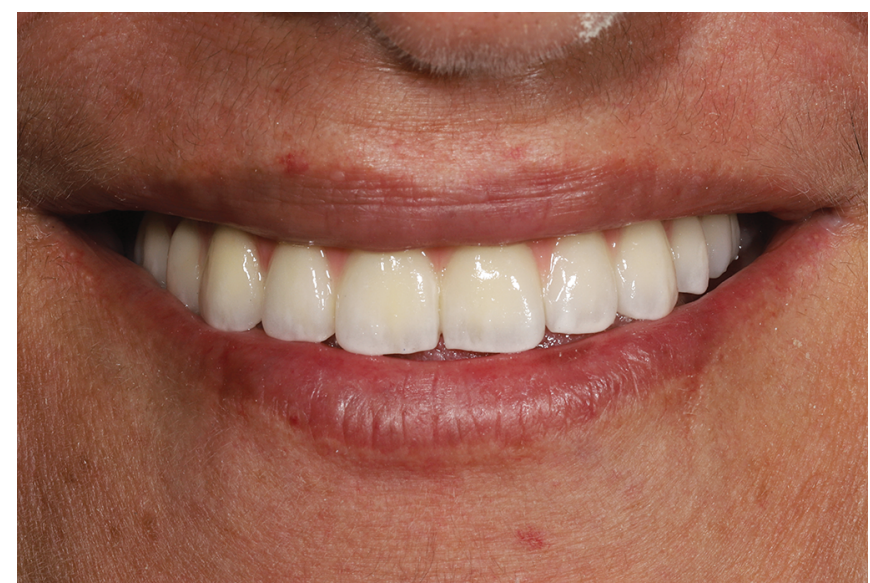

Fig. 6: Patient's smile with the new metal-ceramic prosthesis in place

- Using this software, orient your framework into the most appropriate position, select the processing parameters, and determine the melting strategy. Print the framework with selective laser melting (SLM) additive technology (Mysint 100; Sisma SpA, Piovene Rochette, Italy) using a powdered cobalt-chromium alloy (Mediloy S-Co; BEGO Medical GmbH, Bremen, Germany) at a speed of $800 \mathrm{~mm} /$ minute and a layering thickness of $20 \mu \mathrm{m}$. After additive manufacturing, heat treats the framework at $800^{\circ} \mathrm{C}$ for 15 minutes.

- Separate the framework from its supports and subject it to air particle abrasion with $110 \mu \mathrm{m} \mathrm{Al}_{2} \mathrm{O}_{3}$ particles at $0.4 \mathrm{MPa}$ and finish in one direction with carbide burs (Cross Cut; Sigmadent, Istanbul, Turkey).

- Unscrew the old prosthesis and insert the metal framework intraorally. Evaluate passive fit using the one screw test once again (Fig. 5).

- Register the maxillomandibular relationship to conventionally mount the maxillary plaster cast and the mandibular printed cast using PVS bite registration material (Occlusofast+; Zhermack, Badia Polesine, Italy).

- Print the STL file of the mandibular arch (ProJet; 3D Systems Inc., South Carolina, USA) and articulate it with the maxillary plaster cast on a semi-adjustable articulator of your choice.

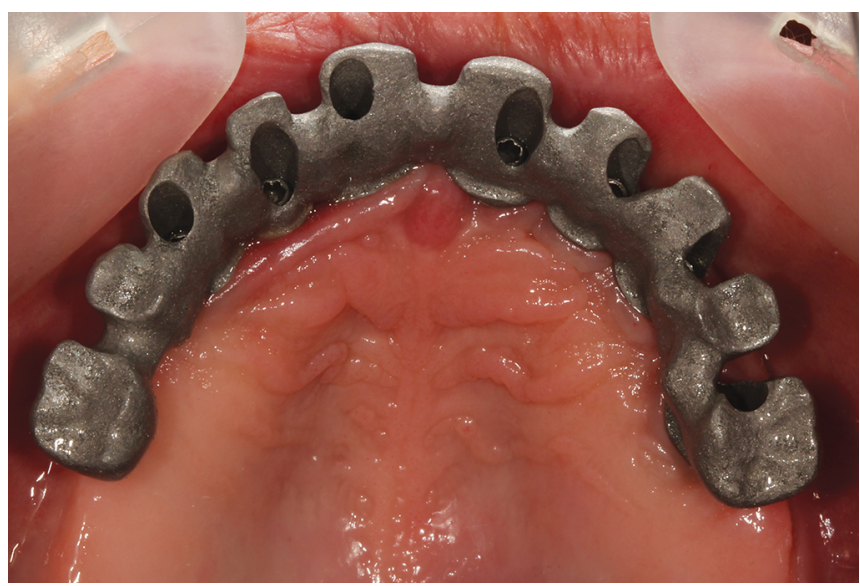

Fig. 5: Intraoral try-in of the metal framework to assess passive fit before ceramic layering

- Layer white and pink ceramics on the metal framework to simulate natural hard and soft tissue to achieve esthetic outcomes (Creation; Creation Willi Geller $\mathrm{GmbH}$, Meiningen, Austria). Sinter the ceramic at a firing temperature of $940^{\circ} \mathrm{C}$.

- Unscrew the old prosthesis and insert the new one for try-in to re-verify the ideal fit with the implants. Evaluate and confirm esthetics and occlusion before glazing the prosthesis at a temperature of $880^{\circ} \mathrm{C}$.

- Unscrew the old prosthesis for the last time and torque the final implant level metal-ceramic prosthesis to the value recommended by the manufacturer. Seal the screw access holes with PTFE tape, followed by composite resin (Filtek Supreme Ultra Universal Restorative; 3M, Minnesota, USA) (Fig. 6).

\section{Discussion}

Transforming an existing metal acrylic hybrid to a layered ceramic hybrid often necessitates going through the entire fabrication process from scratch, including making new impressions with impression copings and verification jigs to check the accuracy of the impressions and to detect incidences of misfits. ${ }^{14}$ This is often considered time-consuming, especially if it has already been done once before. The technique allows the clinician to utilize the existing information and extrapolate it to fabricate a new prosthesis. Fabricating a repositioning impression using an already existing prosthesis allows the restorative dentist to register the ideal spatial implant positioning without the need for a verification jig as the prosthesis is already in optimum fit with the implants. The practitioner may also discard the use of a tray because the hybrid, being reinforced with a metal bar, is not affected by the dimensional changes of the material or by impression removal distortion. Therefore, the uniform distribution of the material and rigidity of the polyvinyl siloxane material is not a concern, even if a tray was not used. In addition, the incorporation of the digital workflow is advantageous as it no longer necessitates timeconsuming procedures and appointments such as the need for record base fabrication, setting of teeth, and forming a silicone index to check the available space for ceramics. The "scan only" files were sufficient for the dental technician to design their fullcontour prosthesis and to perform the necessary reduction to create the metal framework. 
A thorough examination of the existing acrylic prosthesis in terms of occlusal vertical dimension, function, and esthetics is of paramount importance as it will determine the success of the newly fabricated prosthesis. It is also noteworthy to mention that the intaglio surface of the prosthesis should be in intimate contact with the tissue. If a gap is detected, light-body addition silicone must be injected into this space to re-create this intimate contact between the prosthesis and the soft tissue before fabricating the putty silicone matrix for prosthesis pick-up.

The technique presented has multiple limitations that should be considered. The initial chairside time is quite significant as the patient must wait for the setting of the cast before they can receive their prosthesis back after it has been retrieved from the poured pick-up matrix. Furthermore, the cost for fabricating a metal bar and layering it with ceramic by a master ceramist may be significant in some regions like North America, making this option difficult to pursue financially for both the patient and the restorative dentist. With the advancement of digital technology and dental materials, pre-processed milled PMMA and monolithic zirconia hybrids are being fabricated and used more often and may eventually make hand-made prostheses like this one mute, as the lab technicians can easily re-mill the digitally designed prosthesis if complications occur. However, although this technique was done to convert acrylic to metal-ceramic, the same principles could theoretically apply for conversions from acrylic to monolithic zirconia hybrid prostheses, making it a useful approach regardless of the end restorative material.

Overall, the use of this technique has resulted in a significantly reduced number and length of appointments in comparison with the conventional impression technique making it highly favored as a treatment approach for both the patient and the restorative dentist. Future research to compare and overlay the three-dimensional positioning and angulation of the implant analogs between the new and old prostheses may be beneficial to validate this technique due to the well-established limitation and subjectivity of clinically determining complete passive fit.

\section{Conclusion}

This article describes a combined analog and digital workflow for converting an acrylic hybrid to a metal-ceramic prosthesis with immediate passive fit through a pick-up impression. This reduces overall chairside and appointment time and provides a predictably fitting prosthesis.

\section{ACKNOWLedgments}

The authors thank Details Lab by Jean Noujeim, Mr Roland Noujeim, and Mrs Aline Abou Faical for the design and lab work on this case.

\section{References}

1. Elsyad MA, Elgamal M, Mohammed Askar O, et al. Patient satisfaction and oral health-related quality of life (OHRQoL) of conventional denture, fixed prosthesis and milled bar overdenture for all-on- 4 implant rehabilitation. A crossover study. Clin Oral Implants Res 2019;30(11):1107-1117. DOI: 10.1111/clr.13524.

2. Oh SH, Kim Y, Park JY, et al. Comparison of fixed implant-supported prostheses, removable implant-supported prostheses, and complete dentures: Patient satisfaction and oral health-related quality of life. Clin Oral Implants Res 2016;27(2):1-7. DOI: 10.1111/clr.12514.

3. Goodacre C, Goodacre B. Fixed vs removable complete arch implant prostheses: a literature review of prosthodontic outcomes. Eur J Oral Implantol 2017;10:13-34.

4. Jivraj S, Chee W, Corrado P. Treatment planning of the edentulous maxilla. Br Dent J 2006;201(5):261-279. DOI: 10.1038/sj.bdj. 4813952.

5. Wicks RA. A systematic approach to definitive planning for osseointegrated implant prostheses. J Prosthodont 1994;3(4):237242. DOI: 10.1111/j.1532-849x.1994.tb00161.x.

6. Mertens C, Steveling HG. Implant-supported fixed prostheses in the edentulous maxilla: 8-year prospective results. Clin Oral Implants Res 2011;22(5):464-472. DOI: 10.1111/j.1600-0501.2010.02028.x.

7. Heydecke G, Zwahlen M, Nicol A, et al. What is the optimal number of implants for fixed reconstructions: a systematic review. Clin Oral Implants Res 2012;23:217-228. DOI: 10.1111/j.1600-0501.2012. 02548.x.

8. Gallucci GO, Doughtie CB, Hwang JW, et al. Five-year results of fixed implant-supported rehabilitations with distal cantilevers for the edentulous mandible. Clin Oral Implants Res 2009;20(6):601-607. DOI: 10.1111/j.1600-0501.2008.01699.x.

9. Meng GK, Chung KH, Fletcher-Stark ML, et al. Effect of surface treatments and cyclic loading on the bond strength of acrylic resin denture teeth with autopolymerized repair acrylic resin. J Prosthet Dent 2010;103(4):245-252. DOI: 10.1016/S0022-3913(10) 60038-8.

10. Purcell BA, McGlumphy EA, Holloway JA, et al. Prosthetic complications in mandibular metal-resin implant-fixed complete dental prostheses: a 5- to 9-year analysis. Int J Oral Maxillofac Implants 2008;23(5):847-857.

11. Bozini T, Petridis H, Garefis K, et al. A meta-analysis of prosthodontic complication rates of implant-supported fixed dental prostheses in edentulous patients after an observation period of at least 5 years. Int J Oral Maxillofac Implants 2011;26(2):304-318.

12. Gonzalez-Gonzalez I, deLlanos-Lanchares H, Brizuela-Velasco A, et al. Complications of fixed full-arch implant-supported metal-ceramic prostheses. Int J Environ Res Public Health 2020;17(12):4250. DOI: 10.3390/ijerph17124250.

13. Tischler M, Bagley D. Acrylic hybrid denture converted to monophase zirconia. Inside Dentistry 2014;10.

14. Ercoli C, Geminiani A, Feng C, et al. The influence of verification jig on framework fit for nonsegmented fixed implant-supported complete denture. Clin Implant Dent Relat Res 2011; 14:188-195. DOI: 10.1111/j.1708-8208.2011.00425.x. 